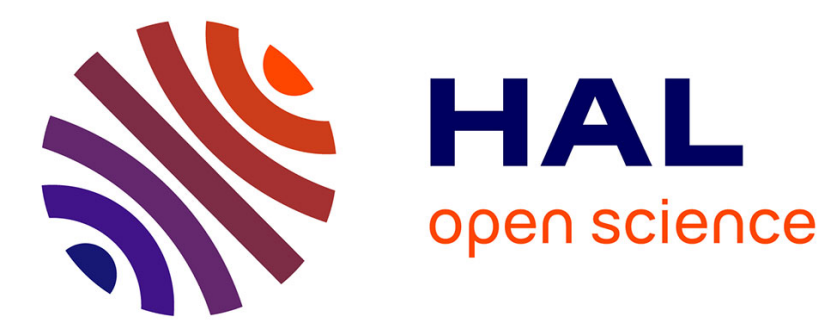

\title{
Taking into account geometrical variation effect on product performance
}

Dinh Son Nguyen, Frédéric Vignat, Daniel Brissaud

\section{To cite this version:}

Dinh Son Nguyen, Frédéric Vignat, Daniel Brissaud. Taking into account geometrical variation effect on product performance. International Journal of Product Lifecycle Management, 2011, 5 (2-3), pp.102-121. 10.1504/IJPLM.2011.043183 . hal-00802354

\section{HAL Id: hal-00802354 \\ https://hal.science/hal-00802354}

Submitted on 27 Mar 2013

HAL is a multi-disciplinary open access archive for the deposit and dissemination of scientific research documents, whether they are published or not. The documents may come from teaching and research institutions in France or abroad, or from public or private research centers.
L'archive ouverte pluridisciplinaire HAL, est destinée au dépôt et à la diffusion de documents scientifiques de niveau recherche, publiés ou non, émanant des établissements d'enseignement et de recherche français ou étrangers, des laboratoires publics ou privés. 


\title{
Taking into account geometrical variation effect on product performance
}

\section{Dinh Son Nguyen*, Frédéric Vignat and Daniel Brissaud}

\author{
G-SCOP Laboratory,
} Grenoble Institute of Technology,

46 Avenue Félix Viallet, Grenoble 38031, France

Fax: (33)-4-76-57-46-95

E-mail: dinh-son.nguyen@g-scop.inpg.fr

E-mail: nguyen.dson@gmail.com

E-mail: frederic.vignat@g-scop.inpg.fr

E-mail: daniel.brissaud@g-scop.inpg.fr

*Corresponding author

\begin{abstract}
Due to rapid development of technology and strict competition in the context of global and concurrent economy, the requirements of customers such as quality, reliability, sustainability and cost of products are more and more high and tight. Thus, satisfaction of those is an important key of product designers. However, the product designers work mainly on the nominal model of the product within a CAD/CAM system. These models can only represent nominal information about the product and have no ability to deal with various kinds of deviations during the product life cycle. These deviations can make the designed product not to meet fully the requirements of the customers. Thus, it is necessary to take into account the geometrical deviations to simulate the 'real' performances of the product. In this paper, we propose a method to integrate the geometrical deviations of the product into 'real' performance simulations. As a result, the product designers can generate the performance of the expected population of 'real' products. They can verify that the product they are designing will have 'real' performances satisfying the requirements of customers and users.
\end{abstract}

Keywords: life-cycle engineering; manufacturing; product geometrical deviations; product lifecycle engineering; geometrical variation effect; product performance.

Reference to this paper should be made as follows: Nguyen, D.S., Vignat, F. and Brissaud, D. (2011) 'Taking into account geometrical variation effect on product performance', Int. J. Product Lifecycle Management, Vol. 5, Nos. 2/3/4, pp.102-121.

Biographical notes: Dinh Son Nguyen received his MS in Industrial Engineering from the Grenoble Institute of Technology in 2007. He is currently working as a PhD student under the guidance of Professor Daniel Brissaud and Assistant Professor Frédéric Vignat in integrated design team of the Laboratory G-SCOP at the Grenoble University. His research interests are modelling geometrical deviations of product, simulation of manufacturing processes and robust design methodology. 
Frédéric Vignat obtained his $\mathrm{PhD}$ from the Grenoble Institute of Technology in 2005. He is actually an Associate Professor at the University of Grenoble (Grenoble-INP). He is working in the Laboratory G-SCOP of the Grenoble University in the area of integrated design. His research interests include manufacturing simulation, product tolerancing and life cycle engineering. $\mathrm{He}$ has published more than 20 papers in these areas in international journals and conferences. He mentored many Masters thesis and two PhD theses.

Daniel Brissaud has been a Professor of Engineering Design and Eco-Design at the University of Grenoble (Grenoble-INP) since 1998. He is the Head of the Cluster of Research 'Management and Organisation of Production Systems and Innovation' for the Rhône-Alpes area. He is a member of the European Manufacturing and Innovation Research Association 'EMIRAcle', and CIRP Fellow. He was the Head of the Doctoral studies in Industrial Organisations and Production Systems. His scientific interests are eco-design, environmental assessment, life cycle engineering, clean technologies, product-service systems design, integrated design and requirement engineering. He has authored more than 60 papers in international journals and books and mentored $16 \mathrm{PhD}$ theses.

This paper is a revised and expanded version of a paper entitled 'Product performance simulation with geometric deviations throughout its life cycle' presented at The 6th CIRP-Sponsored International Conference on Digital Enterprise Technology, University of Hong Kong, Hong Kong, 14-16 December 2009.

\section{Introduction}

Owing to the development of information technology, computers are becoming a useful and effective tool supporting most of the engineering activities in product design and manufacturing. Most of the product designers create a numerical model of a product by a number of computer aided tools, such as $\mathrm{CAD} / \mathrm{CAM}$, engineering simulation, product data management (PDM) and product life cycle management (PLM). However, the product model created in this environment is a nominal representation that does not take into account variations generated along the product life cycle, such as geometrical deviations generated by manufacturing and assembly processes.

The product must, in fact, pass through many stages of its life cycle before arriving in the hand of the customer and of the users. Each part making up the product is manufactured from raw material by the manufacturing processes (forging, cutting, grinding, etc.). Geometrical deviations on each part are generated and accumulated over the successive set-up of the multistage manufacturing process due to imperfections of material, tooling and machine. The parts with deviations are then assembled at the assembly stage. The surfaces' deviations of each part have not only an influence on the assemblability but are also accumulated on the product in the assembly stage. The product geometry is, therefore, different from the nominal one at the end of the manufacturing and assembly stages.

The current product modelling technology presented above is, unable to take into account these deviations. Most of the simulations to predict the behaviour of the product (kinematics, dynamics, resistance, fluid flow, etc.) are carried out based on the nominal model of the product. Thus, the result of the performance simulation of the designed product can be considered as nominal and consequently different from the real one. The 
risk is then that the designed product does not meet fully the requirements of the customers and users. In this case, the product-process design has to be considered as unsuitable or at least not to be robust.

There are, therefore, important issues that require to be considered:

- How to model the geometrical deviations of a product generated throughout its life cycle?

- How to manage the causes and consequences of these deviations in the design stage?

- How to simulate the performance with geometrical deviations of the product?

Some answers exist today in the academic research for each stage of the product life cycle separately. There is, however, no complete answer for all stages yet. Thus, we propose, in this paper, a method that allows to model the geometrical deviations of the product throughout all stages of its life cycle and integrating these deviations into the performance simulation of the product. The aim is to predict the real performance of the product taking into account the geometrical deviations. As a result, product designers can verify that the product they are designing will have real performances satisfying the requirements of customers and users.

\section{Literature review}

\subsection{Manufacturing stage}

Many researches for modelling geometrical deviations generated during manufacturing stage have been done. They can be classified into two different approaches. The first one is based on the concept of state space model. The models to describe dimensional variation propagation along multistage machining processes using this approach are proposed by Zhou et al. (2003) and Huang et al. (2003). A state vector $x(k)$ is used to describe workpiece deviations at $k$ th stage. These deviations are accumulated and transformed onto the workpiece during previous stages $(1 \ldots k-1)$ of the multistage machining process. The set of state vectors $x(k)$ describes the workpiece deviations relative to nominal one resulting of the whole machining processes. This model provides a quantitative relationship between the fixture locator errors and the final workpiece geometrical error and has great potential to be applied to fault diagnosis and process design evaluation for complicated machining processes.

The second approach is based on the concept of small displacement torsor (SDT) that is proposed by Bourdet et al. (1996). The method based on the SDT approach to perform 3D manufacturing tolerancing for mechanical parts is, firstly, proposed by Villeneuve et al. (2001). Thereafter, a model of manufactured parts (MMP) based on the SDT for simulating and storing the manufacturing defects in 3D has been developed by Vignat and Villeneuve (2007). It permits to collect the deviations generated during a virtual manufacturing process. The defects generated by a machining process are considered to be the result of two independent phenomena: the positioning and the machining deviations accumulated over the successive set-ups. The positioning deviation is the deviation of the nominal part relative to the nominal machine. The positioning operation of the part on the part-holder is realised by a set of hierarchically organised elementary connections. The manufactured deviations of surface relative to its nominal 
position in MMP are expressed by the parameters of the SDT. Tichadou et al. (2005) proposed a graph representation of the manufacturing process. This graph model the successive set-up and for each set-up the positioning surface and their hierarchy and the machined surfaces. This graph makes it possible to highlight the influential paths. They propose then two analysis methods. The first one is based on the concept of SDT. The second one is based on the use of CAD software in which they model a manufacturing process with defects. They then virtually measure the realised part and check its conformity.

\subsection{Assembly stage}

A product is made up of parts assembled by the way of connections. Each part has already passes through the manufacturing stage where geometrical deviations have been generated. Then the product passes through assembly stage of its life cycle. Assembly stage of the product life cycle is an essential stage, and it obviously brings its share of deviations to the product.

In general, models for mechanical assembly can be categorised into two different approaches. The first one is based on the state space model. Some models representing this approach are proposed by Mantripragada and Whitney (1999) and Huang et al. (2007) with stream-of-variation model (SOVA) for 3D rigid assemblies dimensional variation propagation analysis in multi-station processes. The deviations accumulated at $i$ th assembly station are described by a vector $X(i) \in R^{n_{t} \times 1}$. The state space model of a multistage assembly process is represented by equation (1).

$$
\left\{\begin{array}{l}
X(i)=A(i-1) X(i-1)+B(i) U(i)+W(i) \\
Y(i)=C(i) X(i)+V(i)
\end{array}\right.
$$

where $U(i) \in R^{m(i) \times 1}$ is the fixture/part deviation contribution from station $i$; $Y(i) \in R^{q(i) \times 1}$ is the measurement obtained on station $i ; W(i)$ and $V(i)$ are mutually independent noise and $A(i), B(i)$ and $C(i)$ are transformation matrix.

The second approach is based on the SDT concept. Bourdet and Ballot (1995) introduced the geometrical behaviour laws based on the SDT for modelling geometrical deviations in the mechanism. Thereafter, Thiebaut (2001) proposed a model to analyse part deviation in assembly. The positioning variation of the part relative to its nominal position in the global coordinate system is expressed by equation (2).

$$
D(A / R)=E\left(A / S_{A}\right)+T\left(S_{A} / S_{B}\right)+E\left(S_{A} / B\right)+D(B / R)
$$

where

$D(A / R) \quad$ is the variation of part $A$ relative to its nominal position in global coordinate system.

$E\left(A / S_{A}\right) \quad$ is the variation of surface $S_{A}$ of the part $A$ relative to its nominal position.

$T\left(S_{A} / S_{B}\right) \quad$ is the variation of link between the surface $\mathrm{S}_{\mathrm{A}}$ of the part $A$ and the surface $S_{B}$ of the part $B$.

$E\left(S_{A} / B\right) \quad$ is the variation of surface $S_{B}$ of the part $B$ relative to its nominal position.

$D(B / R) \quad$ is the variation of part $B$ relative to its initial nominal position in global coordinate system. 
A linear system of equations is created from the contribution of each connection between part $A$ and part $B$. The positioning variation of part $A$ is determined by resolution of the linear system of equations based on Gauss-elimination method.

The main principle of the models proposed by Mantripragada and Whitney (1999), Huang et al. (2007) and Thiebaut (2001) is to model the variation of the part at each stage along the assembly process. However, they do not link the geometrical deviations of the surfaces of the parts to the parameters of the manufacturing process. These parameters are those of the MMP (Vignat and Villeneuve, 2007) that can be measured from the manufacturing process as proposed by Tichadou et al. (2007). That is the reason why the authors have already proposed a model using the SDT in order to model geometrical deviations generated during the manufacturing stage and accumulated in the assembly stage.

\subsection{Design stage}

The geometrical deviations generated and accumulated during the product life cycle affect the performance of the product. It is thus necessary to manage their causes and consequences at the design stage in order to reduce their effect on the product performance all along its life cycle.

Parkinson (1995) proposed to use engineering models for developing robust design in order to reduce the variance of the design under variation of sources, such as manufacturing operations, variation in material properties, and operating environment. $\mathrm{Yu}$ and Ishii (1998) addressed the impact of the manufacturing errors on the performance of the product. He defined the manufacturing variation pattern (MVP) to represent the manufacturing characteristics and investigated its effects on the performance of the product. Zhu and Ting (2001) presented the theory that offers the analytical and geometrical description of the performance sensitivity distribution of a product in the variation space. The theory can be applied to find the robust design less sensitive to the dimensional variation due to manufacturing errors or product wear. Liu and Chen (2006) proposed a new probabilistic sensitivity analysis (PSA) approach for design under uncertainty based on the concept of relative entropy. This approach allows to provide the valuable information about the impact of the design variables on the performance of the product and whole range or a partial range of the performance distribution. Bruyère et al. (2007) proposed a statistical approach in order to evaluate the impact of the geometrical variations on the angular rotational velocity between two bevel gears. Monte-Carlo simulation method is used to consider the geometrical behaviour simulation and tooth contact analysis. Yin et al. (2009) proposed to integrate material and manufacturing process uncertainties in the design in order to consider their impacts on the performance of the product. They developed a procedure for uncertainty propagation from the material random field to the end product performance based on the product finite-element mesh.

These studies examine the impact of the variation sources or the geometrical variations on the product performance variation. Most of the relationship approximations between the performance and the design variables are only based on the nominal model of the product. Thus we propose, in this paper, a method that allows to model the geometrical deviations of product during its life cycle and integrating them into the performance simulation. The aim is to manage the geometrical variability throughout the product life cycle and their impacts on the performance. 


\section{Integration of geometrical deviations into the performance simulation of a product}

The numerical model of product created in CAD/CAM systems is usually used to approximate the practical performance of the product. The approximation can make performance of the designed product less precise in comparison with the customers' requirements because there are many variation sources that affect the product performance in the real environment.

Figure 1 The overview of method (see online version for colours)

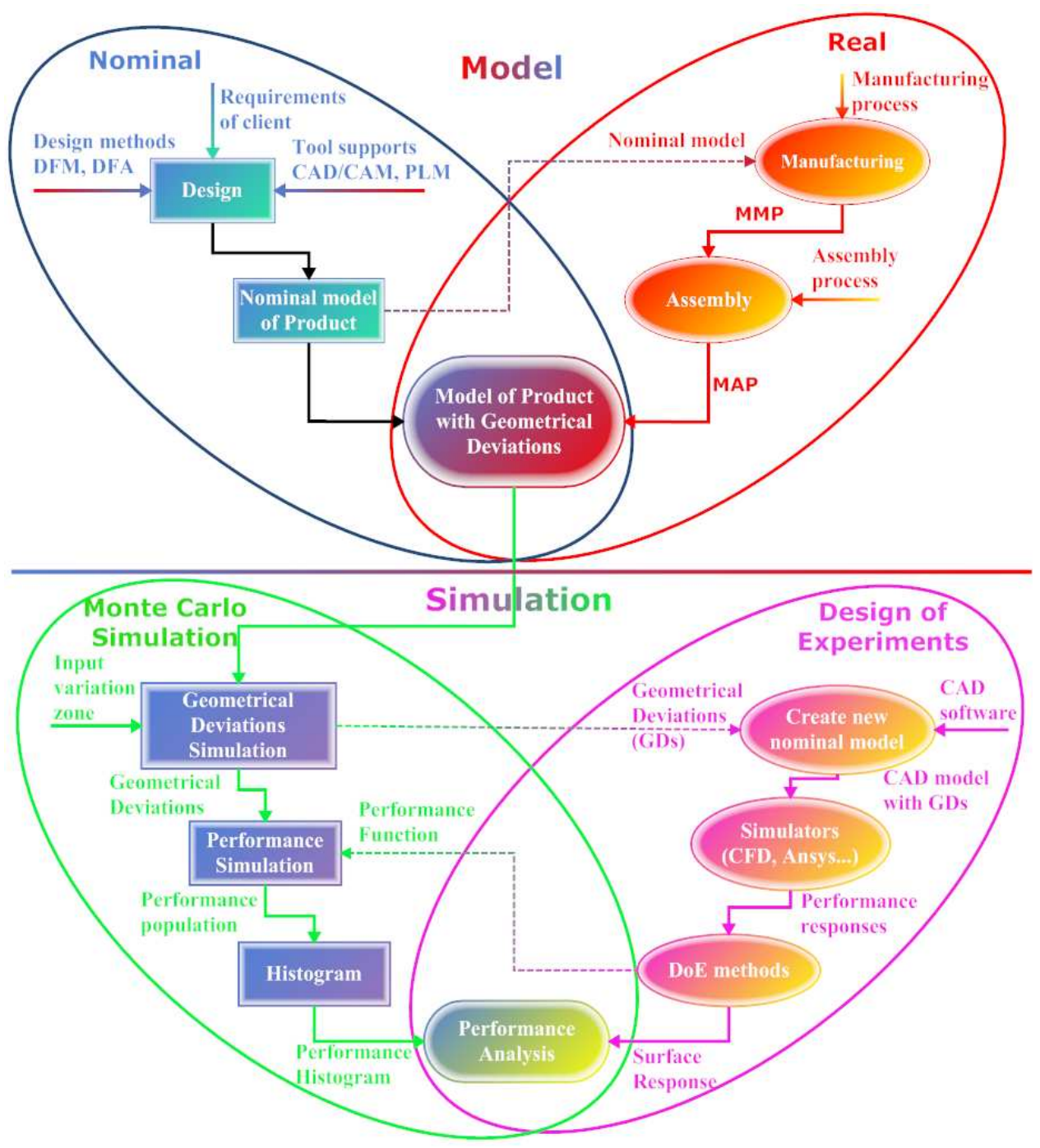


Kimura (2007) mentioned the existence of disturbance in the total product life cycle, such as material property defects, manufacturing process errors, assembling process inaccuracy and mal-operations in product usage stages, etc. They may influence the product quality and functional failure. He also proposed a reliability design method to predict product behaviour under these disturbances and to verify product functionality throughout the product life cycle. However, this method only relates to functional failure analysis not to geometrical variability analysis. Thus, we propose, in this paper, a method that allows to model the geometrical deviations and to integrate them into the performance simulation. An overview of the method is described by Figure 1. It is separated in two distinct branches. The first one consists in the geometrical deviation model generated in the manufacturing and assembly stage, and the second one includes the simulation of the performance of the product taking into account these deviations.

The geometrical deviations generated and accumulated during the manufacturing and assembly processes are modelled by the MMP and the model of assembled part (MAP). These models are presented in the next chapter. Monte-Carlo simulation method is used to evaluate the geometrical variations of the product. The relationship between the product performance and the parameters of the variation sources is established by using the design of experiment method. The distribution of the population of the product performance is then calculated in order to verify of the performance variation in comparison with the customers requirements.

\subsection{Geometrical deviation model of a product}

The geometrical deviation model of the product during the manufacturing and assembly stage has been presented in detail by Nguyen et al. (2010). For manufacturing stage, the deviation of surface $j$ of manufactured part $i$, manufactured in set-up $S j$, relative to its nominal position is considered to be the result of two independent phenomena: the positioning and the machining deviations accumulated over the successive set-ups, as expressed by equation (3).

$$
T_{P^{i}, P_{j}^{i}}=-T_{S j, P^{i}}+T_{S j, P_{j}^{i}}
$$

$T_{S j, P_{j}^{i}}$ is a SDT modelling the deviation of the machined surface $j$ realised in set-up $S j$ relative to the nominal machine. This torsor merges deviations of the surface swept by the tool and cutting local deformations. $T_{S j, P^{i}}$ is a SDT modelling the positioning deviation of workpiece $i$ in set-up $S j$.

For assembly stage, the geometrical deviations accumulated on the product are modelled by the MAP based on the gap torsor concept proposed by Bourdet et al. (1996). The geometrical deviation of surface $j$ of part $i$ relative to the global coordinate system is expressed by equation (4).

$$
T_{P, P_{j}^{i}}=T_{P, P^{i}}+T_{P^{i}, P_{j}^{i}}
$$

where $T_{P^{i}, P_{j}^{i}}$ is SDT modelling the deviation of surface $j$ of part $i$ relative to its nominal position in the local coordinate system of the part $i$. It comes from the manufacturing stage. $T_{P, P^{i}}$ is a SDT modelling the positioning deviations of part $i$ relative to its nominal 
position in the global coordinate system of the product. The determination of this torsor is based on a set of elementary connection between part $i$ and others, as explained in detail by Nguyen et al. (2010).

In conclusion, the whole geometrical deviations of all the surfaces of the product relative to their nominal positions in the global coordinate system of product will be collected by a set of SDT $T_{P, P_{j}^{i}}$.

\subsection{Simulation of product deviation generation}

In order to integrate geometrical deviations into the simulation of the product performance, it is necessary to have a good image of the real production. There are many methods to study geometrical deviations variation, such as method of moments for estimating its characteristics as mean, variance, median, etc.; bootstrap for approximating the distribution of the population; first/second order reliability method (FORM/SORM) to consider the geometrical reliability of the product (Ballu et al., 2008); Monte-Carlo simulation methods to study systems with a large number of coupled degrees of freedom, such as fluids, disordered materials, strongly coupled solids, and cellular structures. In the current case, Monte-Carlo simulation method can thus be considered as the most effective tool to give an overall image based on the relationship between geometrical deviations and parameters of variation sources (parameters representing part-holder quality, machining/tool defects, etc.) established by the geometrical deviation model. It will be used to predict the amount of variations generated and accumulated throughout the product life cycle. This is the second step of the proposed method. It is to simulate geometrical deviations by using a Monte-Carlo method to generate an image of the real production with geometrical deviations. To this aim, the population of products is virtually manufactured by the following steps:

\section{Step 1 Define the probability distribution type or the variation zone of the input variables}

The input variables are the parameters of the torsors representing the quality of the manufacturing fixtures and the capabilities of the machine tool. The determination of the distribution of these variables is based on experimental measurements or manufacturing process knowledge (Tichadou et al., 2007). The strategies to mathematically bound variation of the input parameters are presented by Nejad et al. (2009). In the case of a plane, the torsor $T_{S j, H i S j}$ modelling the deviations of the part-holder surface is expressed by equation (5).

$$
T_{S j, H i S j}=\left\{\begin{array}{cc}
r x_{S j} & 0 \\
r y_{S j} & 0 \\
0 & t z_{S j}
\end{array}\right\}_{(O, X, Y, Z)}
$$

The variation zone of each parameter can mathematically be defined by equation (6) where max and min value for each parameter comes from measurement or process knowledge. 


$$
\left\{\begin{array}{l}
r x_{S j \text { min }} \leq r x_{S j} \leq r x_{S j \text { max }} \\
r y_{S j \text { min }} \leq r y_{S j} \leq r y_{S j \text { max }} \\
t z_{S j \text { min }} \leq t z_{S j} \leq t z_{S j \text { max }}
\end{array}\right.
$$

Step 2 Generate randomly a set of input values according to their distributions and variation zone

The set of input values is randomly generated by cellular automata algorithm in relation with the defined distributions and variations zone. The cellular automata have been defined by Wolfram (1983). It is a simple mathematical idealisation of natural systems. This algorithm is available within mathematica software and that is the reason why it has been chosen for this work.

Step 3 Calculate the values of the determined components $(l r, l t)$ of the link deviation torsor of the MMP

The determination is based on the non-penetration constraints and positioning functions. The deviation torsor $T_{P^{i}, P_{j}^{i}}$ of the surface $j$ of the manufactured part $i$ is calculated by replacing the determined parameters of the deviation link torsor.

In the case of floating contact, the determined components are randomly chosen in an interval satisfying the non-penetration constraints. For a cylinder-cylinder connection [see Figure 2(a)], the components of the link torsor are expressed by equation (7).

$$
T_{H i S j, P_{m}^{i}}=\left\{\begin{array}{ll}
l r x_{S j} & l t x_{S j} \\
l r y_{S j} & l t y_{S j} \\
U l r z_{S j} & U l t z_{S j}
\end{array}\right\}_{\{O, X, Y, Z\}}
$$

The determined components must comply with the non-penetration conditions expressed in equation (8). Thus, for their determination, the parameters are randomly generated within the intervals expressed in equation (9) and are accepted if they comply with (8) or rejected in the case of non-compliance.

$$
\begin{aligned}
& \left\{\begin{array}{l}
R_{1}-R_{2}+\sqrt{\left(l t x_{S j}-\frac{H}{2} l r y_{S j}\right)^{2}+\left(l t y_{S j}+\frac{H}{2} l r x_{S j}\right)^{2}} \geq 0 \\
R_{1}-R_{2}+\sqrt{\left(l t x_{S j}+\frac{H}{2} l r y_{S j}\right)^{2}+\left(l t y_{S j}-\frac{H}{2} l r x_{S j}\right)^{2}} \geq 0
\end{array}\right. \\
& \left\{\begin{array}{l}
\left|l r x_{S j}\right| \leq \frac{2\left(R_{1}-R_{2}\right)}{H} \\
\left|l r y_{S j}\right| \leq \frac{2\left(R_{1}-R_{2}\right)}{H} \\
\left|l t x_{S j}\right| \leq R_{1}-R_{2} \\
\left|l t y_{S j}\right| \leq R_{1}-R_{2}
\end{array}\right.
\end{aligned}
$$


where $R_{1}, R_{2}$ and $H$ are the radius of cylinder and the height of cylindrical link respectively.

In the case of slipping contact, the determined components are calculated by maximising the positioning function subject to non-penetration constraints. For the planplan connection [see Figure 2(b)], the components of the link deviation torsor are expressed by equation (10).

$$
T_{H i S j, P_{m}^{i}}=\left\{\begin{array}{cc}
l r x_{S j} & U l t x_{S j} \\
l r y_{S j} & U l t y_{S j} \\
U l r z_{S j} & l t z_{S j}
\end{array}\right\}_{\{O, X, Y, Z\}}
$$

The determined components $(l r, l t)$ are calculated by the procedure (11).

Maximise : $-l t z_{S j}$

Subject to :

$$
\left\{\begin{array}{l}
-\frac{b}{2} l r x_{S j}-\frac{a}{2} l r y_{S j}+l t z_{S j} \geq 0 \\
-\frac{b}{2} l r x_{S j}+\frac{a}{2} l r y_{S j}+l t z_{S j} \geq 0 \\
\frac{b}{2} l r x_{S j}-\frac{a}{2} l r y_{S j}+l t z_{S j} \geq 0 \\
\frac{b}{2} l r x_{S j}+\frac{a}{2} l r y_{S j}+l t z_{S j} \geq 0
\end{array}\right.
$$

\section{Step 4 Calculate the surface deviations of each manufactured part of the product based on the MMPS}

This is simply done by addition of the deviation value to the machining value.

\section{Step 5 Assemble the manufactured part of the product based on verification of the $C A$}

During this step, the determined components of the deviation link torsor are calculated based on verification of the assembly constraint $(C A)$. The determination is realised as for Step 3.

\section{Step 6 Calculate the surface deviations of the product in the global coordinate system}

The surface deviations of the product in the global coordinate system are determined by replacing all input parameters into equation (4). All parameters of the model are collected in the end of this step. Then the procedure will be restarted from Step 1 until the desired number of product is reached.

A set of $m$ virtual products is then produced by Monte-Carlo simulation method. All values of parameters of the model are collected in order to be used for simulation of product performance. 
Figure 2 Floating cylindrical contact, (a) floating cylindrical contact (b) slipping planar contact (see online version for colours)

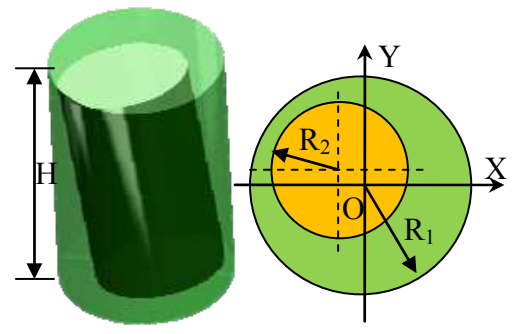

(a)

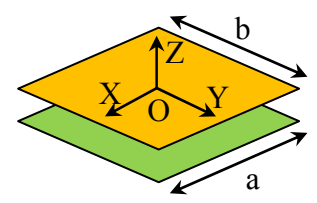

(b)

\subsection{Integration of geometrical deviations into product performance simulation}

The next step is to integrate these deviations into the simulation of product performance. However, it is difficult to integrate all of them into the simulation of the $m$ products, $m$ possibly being one million and each simulation lasting sometime hours (three hours for the computational fluid dynamics (CFD) simulation treated in the next example). Thus a strategy based on design of experiment method to reduce calculation time is proposed in this paper. This method uses factorial design to determine the relationship between the performance and the product geometrical deviations parameters.

\subsubsection{Factorial design}

Factorial design is usually used to understand the effect of two or more independent variables upon a single dependent variable. It is used to study the relationship between the product performance and the geometrical deviations. In order to initiate this method, it is necessary to create an experimental table, called design matrix, which includes factors and their levels. These factors can be the non-geometrical or geometrical deviations parameters. The overview of this method is shown in Figure 3.

Figure 3 Integration of geometrical deviations into product performance simulation(see online version for colours)

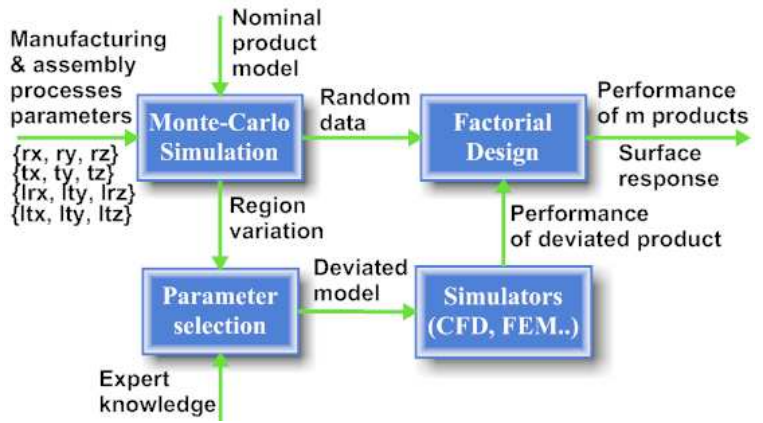

Moreover, it is still time consuming to take all factors because the number of experimental runs depends on the number of factors and the number of levels. $k^{n}$ runs must be realised for $n$ factors and $k$ levels. To reduce the number of factors, key factors 
are defined based on expert knowledge. Then the number of levels for these factors has to be defined depending on a compromise between the desired precision and the calculation time. The value of each level of each factor is calculated according to its range of variation. The $k^{n}$ values of the $n$ parameters are then gathered in a design matrix $P$, as given in equation (12).

$$
P=\left[\begin{array}{cccc}
p_{11} & p_{21} & \ldots & p_{n 1} \\
p_{12} & p_{22} & \ldots & p_{n 2} \\
\vdots & \vdots & \ddots & \vdots \\
p_{1 k^{n}} & p_{2 k^{n}} & \cdots & p_{n k^{n}}
\end{array}\right]
$$

In the simple case, the function performance is known and the performance value is obtained by the value of each parameter $p_{i j}$ of each line of the design matrix into analytical formula. In the complex case, simulation tools as FEA, CFD, etc., need to be used to find the product performance. Thus a set of $k^{n}$ deviated models of the product corresponding to the geometrical parameters $p_{i j}$ has to be created in the CAD system. Each model is used to simulate the product performance in order to determine the response vector $R$ corresponding to the design matrix $\mathrm{P}$, as given in equation (13).

$$
R=\left\{r_{1}, r_{2}, \ldots, r_{k^{n}}\right\}^{T}
$$

The relationship between the product performance and the selected factors is established by the linear or non-linear regression model, as given in equation (14).

$$
\text { Performance }=f\left(p_{1}, p_{2}, p_{3}, \ldots, p_{n}\right)
$$

For example, in the case of $n$ factors and two levels, the function $f$ describing the relationship between the performance and the factors is established from the result of $2^{n}$ simulations, as given in equation (15).

$$
f=\hat{\beta} \cdot p+\varepsilon
$$

where

$p=\left\{p_{1}, p_{2}, p_{3}, \ldots, p_{n}\right\}^{t} \quad$ is a vector of the $n$ factors

$\hat{\beta} \quad$ is a coefficient vector of the model, as calculated by equation (16)

$$
\hat{\beta}=\left(P^{t} \cdot P\right)^{-1} \cdot P^{t} \cdot R
$$

$\varepsilon \quad$ is a residual vector, as calculated by equation (17).

$$
\varepsilon=E(R)=\frac{1}{m} \sum_{i=1}^{m} r_{i}
$$

The performance of the population of products will be generated by replacing the value of the selected factors $\left\{p_{1}, p_{2}, p_{3}, \ldots, p_{n}\right\}$, collected from the Monte-Carlo simulation results, into equation (14). The collection of performance data can then be analysed by using statistic tools. 


\section{Case studies}

In previous chapters, a method to generate an image of the real performance of the product has been proposed. In order to illustrate this method, an example of centrifugal pump design will be presented in this section.

\subsection{A centrifugal pump design}

The numerical model of the centrifugal pump is created in the CAD/CAM software (see Figure 4). In this case, the performance studied is the pump flowrate.

Figure 4 The parts of designed centrifugal pump (see online version for colours)

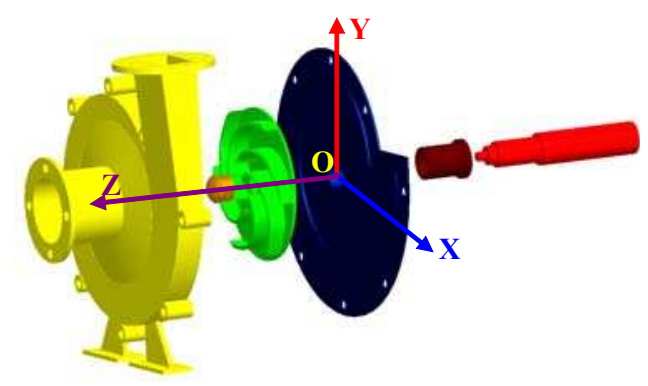

\subsubsection{Geometrical deviation model of the centrifugal pump}

In order to manufacture the pump, manufacturing processes, assembly processes and associated resources are selected according to requirements of customers and cost. The geometrical deviation model of this centrifugal pump during manufacturing and assembly stage is generated by the method as presented in the previous chapter.

Figure 5 Manufacturing process for the shaft of pump (see online version for colours)

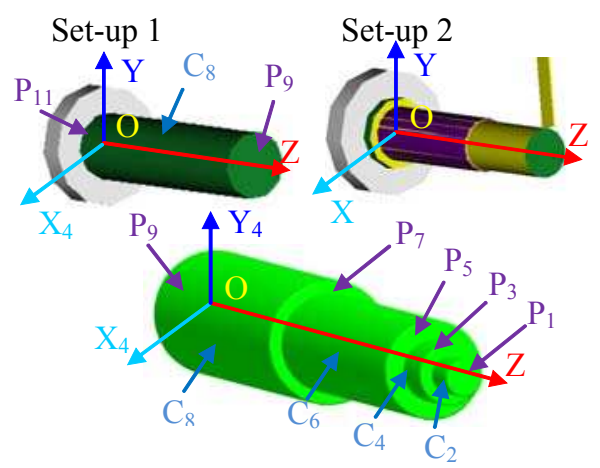

For example, the shaft of the pump is realised by a turning process on a lathe machine (shown in Figure 5). The surface deviations of the planar head of the shaft relative to its nominal position are described by the torsor (18). 


$$
T_{P^{4}, P_{1}^{4}}=\left[\begin{array}{ll}
-l r x_{4,8 S 1}+r x_{4,1}+r x_{4,8}-r x_{4,8 S 1} & 0 \\
-l r y_{4,8 S 1}+r y_{4,1}+r y_{4,8}-r y_{4,8 S 1} & 0 \\
0 & -l t z_{4,9 S 1}+t z_{4,1}+t z_{4,9}-t z_{4,9 S 1}
\end{array}\right]_{\left(O_{4}, R_{4}\right)}
$$

In this torsor, the parameters $r x_{4,1}, r x_{4,8}, r y_{4,1}, r y_{4,8}, t z_{4,1}, t z_{4,9}$ represent the machining inaccuracy and the parameters $r x_{4,8 S 1}, r y_{4,8 S 1}, t z_{4,9 S 1}$ represent the deviations of the fixture surfaces in Set-up 1. The parameters $l r x_{4,8 S 1}, l r y_{4,8 S 1}, l t z_{4,8 S 1}$ represent the link between the fixture surfaces and the surfaces of workpiece in Set-up 1.

The manufactured parts of the pump are then assembled according to the selected assembly process (shown in Figure 6). The geometrical deviations of all surfaces of the pump are described by torsors in the MAP.

Figure 6 Assembly graph of the pump (see online version for colours)

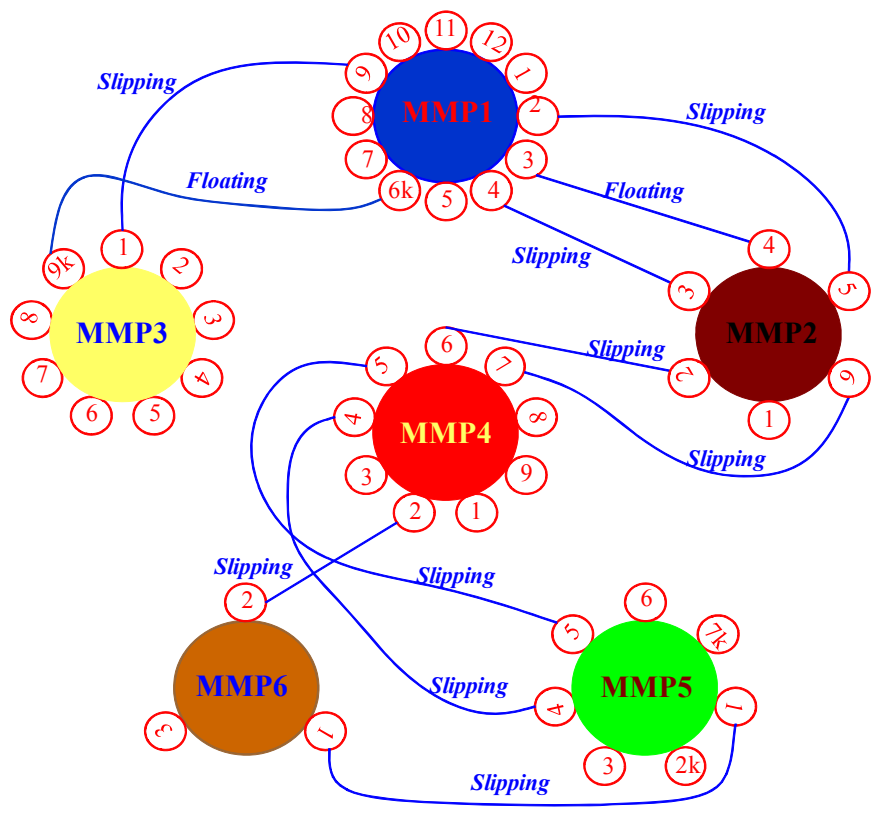

In conclusion, the geometrical deviations of all surfaces of the pump relative to its nominal position in the global coordination system can be modelled according to the selected the manufacturing and assembly processes and the associated resources.

\subsubsection{Monte-Carlo method for geometrical deviation simulation}

The Monte-Carlo simulation method is applied to calculate the values of the geometrical deviations of the pump. The input variables are the parameters of the torsors that represent the machining inaccuracy and the deviation of the fixture surface, such as $r x_{i, j}$, $r y_{i, j}, t x_{i, j}, t y_{i, j}, t z_{i, j}$ for the manufacturing operation of the surface $j$ of the part $i$ and $r x_{i, j s k}$, $r y_{i, j S k}, t x_{i, j S k}, t y_{i, j S k}, t z_{i, j S k}$ for the surface $j$ of the fixture in the set-up $S k$. As explained in the previous chapter, the input variables are considered as independent. The probability distributions and the variation range need to be determined. A uniform distribution is 
chosen in this case. The variation ranges are determined according to the associated resources. For example, the variation ranges of the input parameters for the manufacturing of the shaft are shown in Table 1.

Table 1 Variation range of the input variables

\begin{tabular}{ll}
\hline & \multicolumn{1}{c}{ Set-up 1 } \\
\hline Plane & \multicolumn{1}{c}{ Fixture } \\
4,9S1 & $r x_{4,9 S 1}, r y_{4,9 S 1}$ variation range 0.0001 \\
& $t z_{4,9 S 1}$ variation range 0.02 \\
& $r x_{4,8 S 1}, r y_{4,8 S 1}$ variation range 0.0002 \\
Cylinder & $T x_{4,9 S 1}, t y_{4,8 S 1}$ variation range 0.01 \\
4,8S1 & radius $r a_{4,8 S 1}$ variation range 0.01 \\
\hline & \multicolumn{1}{c}{ Machining } \\
\hline Plan & $r x_{4,5}, r y_{4,5}$ variation range 0.0005 \\
4,5 & $t z_{4,5}$ variation range 0.01 \\
& $R x_{4,4}, r y_{4,4}$ variation range 0.00025 \\
Cylinder & $t x_{44,}, t y_{4,4}$ variation range 0.01 \\
4,4 & $r a d i u s ~ r a_{4,4}$ variation range 0.025 \\
& $R x_{4,7}, r y_{4,7}$ variation range 0.00015 \\
Plan & $t z_{4,7}$ variation range 0.02 \\
\hline
\end{tabular}

One million of pump is virtually manufactured and assembled with deviations by using Monte-Carlo simulation method, as presented above chapter. Then the product designer can use the result to verify any geometrical requirements and to check the assemblability of the pump. For example, the designer can estimate the distribution of the gap between the impeller and the casing of the pump which has to remain positive to avoid contact between impeller and casing. The distribution of the gap is shown in Figure 7(a) (mean $\mu=5.7571 \mathrm{~mm}$, standard deviation $\sigma=0.01976 \mathrm{~mm}$ ). The variation range of the gap according to $6 \sigma$ standard is from $5.6978 \mathrm{~mm}$ to $5.8164 \mathrm{~mm}$.

Figure 7 Monte-Carlo simulation results (see online version for colours)

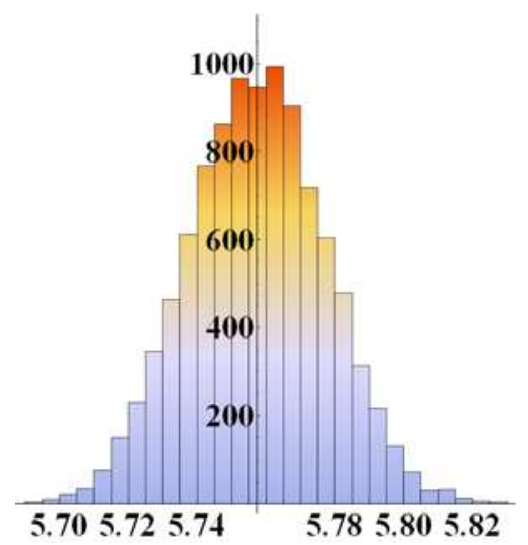

(a)

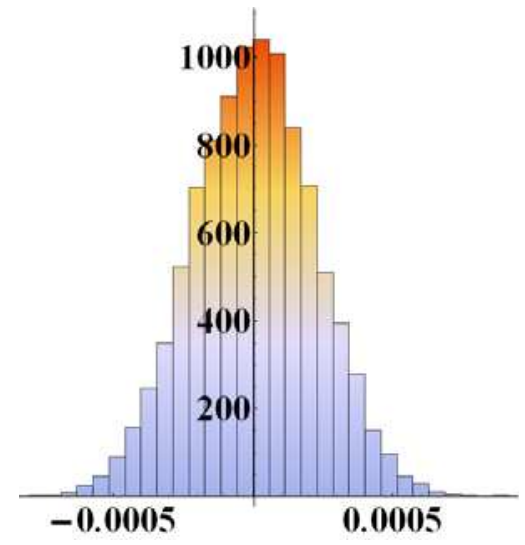

(b) 
Figure 7 Monte-Carlo simulation results (continued) (see online version for colours)

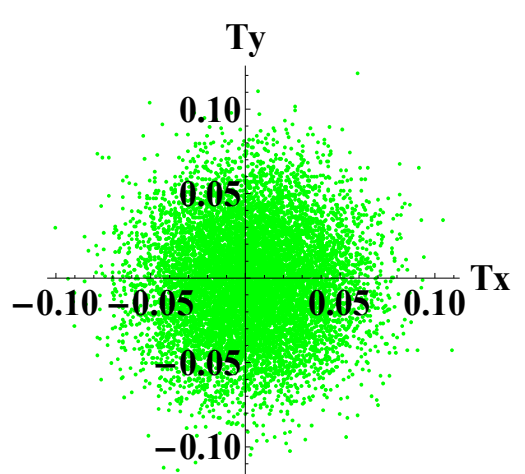

(c)

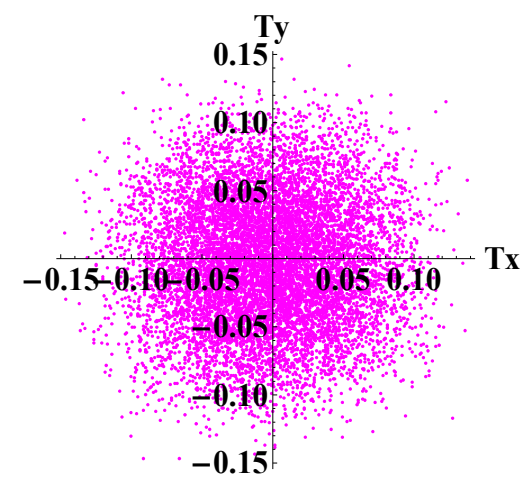

(d)

The designer can also know the distribution of positioning deviation of each part of the pump. For example, the distribution of the gap between the plan of the impeller and the plan of the volute back casing is shown in Figure 7(b). The distribution of the impeller's centre (green colour) and the distribution of the casing's centre (cyan colour) according to two perpendicular axes $\mathrm{X}$ and $\mathrm{Y}$ are shown in Figure 7(b) and 7(c) respectively.

\subsubsection{Design of experiments}

The relationship between the pump flowrate and the geometrical deviations parameters is determined by the factorial design presented above chapter.

Many parameters of the geometrical deviations affect the pump flowrate. It is impossible to integrate all of them into the flowrate simulation. Thus the expert knowledge is used to filter the geometrical deviations parameters that have a strong influence on the pump flowrate. In this example, the gap between the top surface of the impeller and the casing of the pump is selected based on the experiment of Engin and Gur (2001). The translation of the impeller relative to two perpendicular axes $\mathrm{X}, \mathrm{Y}$ in the global coordination system is also chosen based on the study of Baun et al. (2000). Then the value of these three parameters level is calculated from the one million of virtually manufactured pump according to the deviation of the surfaces of the casing and the impeller relative to the global coordinate system of the pump. The value of these deviations is coming from the previous step of the method.

The levels of each factor are then chosen from the data collection of these three factors according to the $6 \sigma$ standard, i.e.:

- $\quad$ high level at $+3 \sigma(1)$

- $\quad$ medium level at $\mu(0)$ (average value of the parameter)

- $\quad$ low level at $-3 \sigma(-1)$.

From the selected factors and levels (see Table 2), 27 deviated models of the pump are created in the CAD software. These are 27 deviated models are used to simulate the pump performance, such as the velocity of fluid, mass flowrate, etc., by using the CFD software. The results concerning the mass flowrate are shown in Table 3. 
Table 2 The value of the selected parameters

\begin{tabular}{lccc}
\hline & Gap $(\mathrm{mm})$ & $T x(\mathrm{~mm})$ & $T y(\mathrm{~mm})$ \\
\hline High level (1) & 5.8164 & 0.08497 & 0.09186 \\
Medium level (0) & 5.7571 & 0.00295 & -0.0027 \\
Low level (-1) & 5.6978 & -0.07907 & -0.09726 \\
\hline
\end{tabular}

Table 3 The results of the flowrate simulation

\begin{tabular}{lccccccccc}
\hline No. & Gap & $T x$ & $T y$ & $Q(g / s)$ & No. & Gap & $T x$ & $T y$ & $Q(g / s)$ \\
\hline 1 & -1 & 1 & 1 & 157975 & 15 & 0 & 0 & -1 & 152688 \\
2 & -1 & 1 & 0 & 157942 & 16 & 0 & -1 & 1 & 158973 \\
3 & -1 & 1 & -1 & 154046 & 17 & 0 & -1 & 0 & 159758 \\
4 & -1 & 0 & 1 & 159899 & 18 & 0 & -1 & -1 & 154705 \\
5 & -1 & 0 & 0 & 159219 & 19 & 1 & 1 & 1 & 153587 \\
6 & -1 & 0 & -1 & 161851 & 20 & 1 & 1 & 0 & 158190 \\
7 & -1 & -1 & 1 & 163015 & 21 & 1 & 1 & -1 & 156502 \\
8 & -1 & -1 & 0 & 158858 & 22 & 1 & 0 & 1 & 164443 \\
9 & -1 & -1 & -1 & 155472 & 23 & 1 & 0 & 0 & 158696 \\
10 & 0 & 1 & 1 & 155809 & 24 & 1 & 0 & -1 & 154314 \\
11 & 0 & 1 & 0 & 158052 & 25 & 1 & -1 & 1 & 145164 \\
12 & 0 & 1 & -1 & 140433 & 26 & 1 & -1 & 0 & 150938 \\
13 & 0 & 0 & 1 & 157969 & 27 & 1 & -1 & -1 & 143754 \\
14 & 0 & 0 & 0 & 156666 & & & & & \\
\hline
\end{tabular}

The relationship between the pump flowrate and the selected parameters is determined by the factorial design method. The mass flowrate $Q$ is a function of the gap between the impeller and the casing of the pump (Gap) and the translation of the impeller relative to two perpendicular axes ( $T x, T y)$, as given in equation (19).

$$
\begin{aligned}
& Q=1.46 \times 10^{7}-5.023 \times 10^{6} \text { Gap }+436656 . \text { Gap }^{2} \\
& +5.970 \times 10^{8} \text { Tx }-2.081 \times 10^{8} \text { Gap Tx }+1.813 \times 10^{7} \text { Gap }^{2} \text { Tx }
\end{aligned}
$$

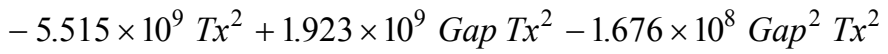

$$
\begin{aligned}
& -2.446 \times 10^{8} T y+8.502 \times 10^{7} \text { Gap Ty }-7.387 \times 10^{6} \text { Gap }^{2} \text { Ty } \\
& \text { + 26906.4 Tx Ty-36301.8 Tx } x^{2} T y+3.44957 \times 10^{9} T y^{2}-1.2 \times 10^{9} \text { Gap Ty }{ }^{2} \\
& +1.036 \times 10^{8} \text { Gap }^{2} T y^{2}-937111 . T x T y^{2}-7.21 \times 10^{7} T x^{2} T y^{2}(g / s) \text {. }
\end{aligned}
$$

Figure 8 shows the associated response surfaces representing the dependence between the mass flowrate of the pump and the factors Gap, $T x$ [Figure 8(a)], the factors Gap, $T y$ [Figure 8(b)] and the factors $T x, T y$ [Figure 8(c)]. 
Figure 8 Response surfaces and distribution of the mass flowrate (see online version for colours)

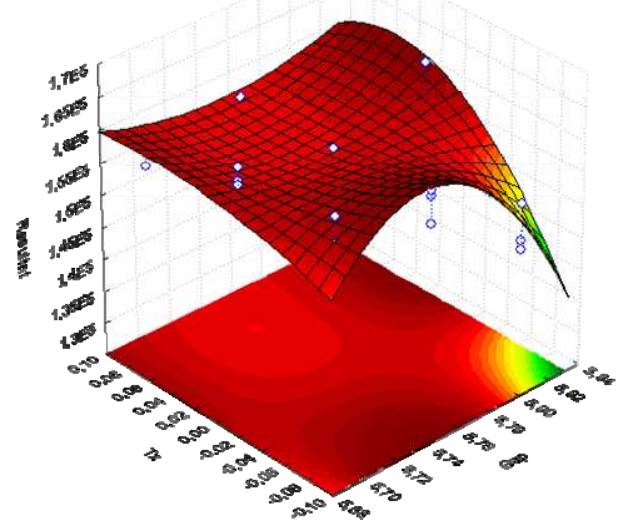

(a)

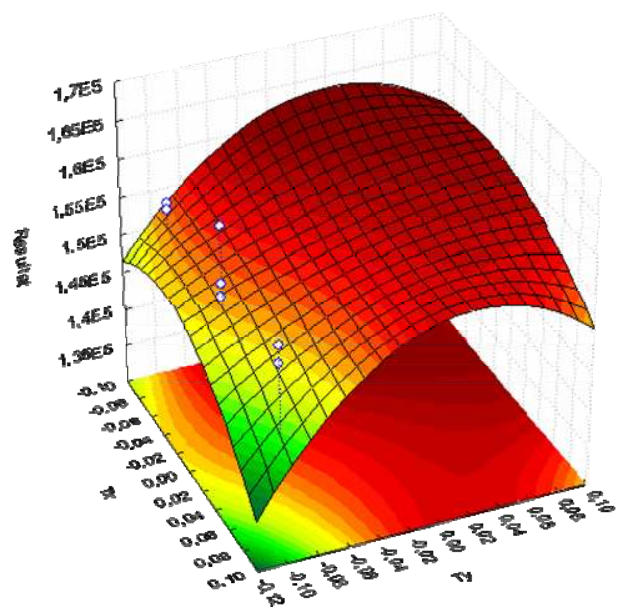

(c)

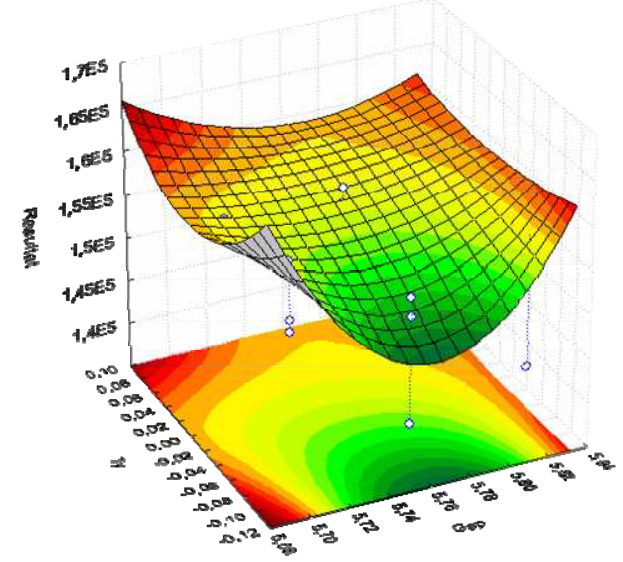

(b)

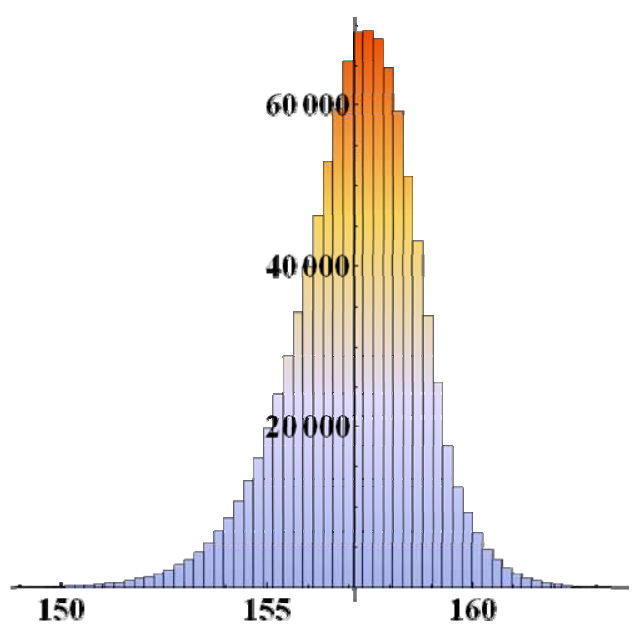

(d)

The product designer then calculates the flowrate of the 1 million pumps based on the values of the Gap, Ty and Ty found in the above session and equation (19). The distribution for the one million of pumps is shown in Figure 8(d). The mean and standard deviation of the mass flowrate of the pump are equal to $157.127 \mathrm{~kg} / \mathrm{s}$ and $1.572 \mathrm{~kg} / \mathrm{s}$ respectively. As a result, the product designer can verify the real mass flowrate of the pump and the satisfaction of customers requirements. Furthermore, he can ensure that the design of the pump plans to deliver to customers is robust.

\section{Conclusions}

Today, the product designers work on the numerical model of the product within a CAD system. This model can only represent nominal product information and most of the 
simulations to predict the behaviour and performance is carried out on this model. Therefore, it limits the ability to deal with geometrical variations generated during the product life cycle. These variations can make the designed product not to meet fully the requirements of the customers and the users.

This paper proposes a method that allows to model the geometrical deviations of the surfaces of the product generated throughout its life cycle and to integrate them into the simulation of the 'real' performance of the product. This method also allows to analyse robustness based on the performance variation. It can thus overcome the weak points of the current product modelling technology by taking into account the geometrical deviations of the product in the performance simulation.

In future work, we will develop this method to deal with the identification of the root cause of performance variation and thus help to make the designed product reliable and robust.

\section{References}

Ballu, A., Plantec, J-Y. and Mathieu, L. (2008) 'Geometrical reliability of overconstrained mechanisms with gaps', CIRP Annals - Manufacturing Technology, Vol. 57, pp.159-162.

Baun, D.O., Köstner, L. and Flack, R.D. (2000) 'Effect of relative impeller-to-volute position on hydraulic efficiency and static radial force distribution in a circular volute centrifugal pump', ASME Journal of Fluids Engineering, Vol. 122, No. 3, pp.598-605.

Bourdet, P. and Ballot, E. (1995) 'Geometrical behavior laws for computer aided tolerancing', Proceedings of the 4th CIRP Seminar on Computer Aided Tolerancing, University of Tokyo.

Bourdet, P., Mathieu, L., Lartigue, C. and Ballu, A. (1996) 'The concept of the small displacement tosor in metrology', Advanced Mathematical Tools in Metrology II, edited by World Scientific Publishing Company, Series Advances in Mathematics for Applied Sciences, Vol. 40, pp.110-122.

Bruyère, J., Dantan, J-Y., Bigot, R. and Martin, P. (2007) 'Statistical tolerance analysis of bevel gear by tooth contact analysis and Monte Carlo simulation', Mechanism and Machine Theory, Vol. 42, pp.1326-1351.

Engin, T. and Gur, M. (2001) 'Performance characteristics of a centrifugal pump impeller with running tip clearance pumping liquid-solid mixtures', ASME Journal of Fluids Engineering, Vol. 123, pp.532-538.

Huang, Q., Shi, J. and Yuan, J. (2003) 'Part dimensional error and its propagation modeling in multi-operational machining processes', Journal of Manufacturing Science and Engineering, Vol. 125, pp.256-262.

Huang, W., Lin, J., Bezdecny, M., Kong, Z. and Ceglarek, D. (2007) 'Stream-of-variation modelling - Part I: a generic three-dimensional variation model for rigid-body assembly in single station assembly processes', Journal of Manufacturing Science and Engineering, Vol. 129, pp.821-831.

Kimura, F. (2007) 'Modeling, evaluation and design of product quality under disturbances throughout the total product life cycle', in Heidelberg, Springer Berlin (Ed.): The Future of Product Development, pp.675-684.

Liu, H. and Chen, W. (2006) 'Relative entropy based method for probabilistic sensitivity analysis in engineering design', Journal of Mechanical Design, Vol. 128, pp.326-337.

Mantripragada, R. and Whitney, E.D. (1999) 'Modeling and controlling variation propagation in mechanical assemblies', IEEE Transactions on Robotics and Automation, Vol. 15, No. 1, pp.124-140. 
Nejad, M.K., Vignat, F. and Villeneuve, F. (2009) 'Simulation of the geometrical defects of manufacturing', The International Journal of Advanced Manufacturing Technology, Vol. 45, Nos. 7-8, pp.631-648.

Nguyen, D.S., Vignat, F. and Brissaud, D. (2010) 'Geometrical deviation model of product throughout its life cycle', International Journal of Manufacturing Research (accepted).

Parkinson, A. (1995) 'Robust mechanical design using engineering models', Journal of Mechanical Design, Vol. 117, pp.48-54.

Thiebaut, F. (2001) 'Contribution à la définition d'un moyen unifié de gestion de la géométrie réaliste basée sur le calcul des lois de comportement des mécanismes', $\mathrm{PhD}$ thesis, ENS de Cachan, France.

Tichadou, S., Kamali Nejad, M., Vignat, F. and Legoff, O. (2007) '3-D manufacturing dispersions: two experimental applications', Proceedings of the 10th CIRP International Seminar on Computer Aided Tolerancing, France.

Tichadou, S., Legoff, O. and Hascoet, J.Y. (2005) '3D geometrical manufacturing simulation: compared approaches between integrated CAD/CAM systems and small displacement torsor models', in D. Brissaud, A. Bramley, D. Coutellier and C. McMahon, (Eds.): Advances in Integrated Design and Manufacturing in Mechanical Engineering, pp.201-214, Springer Netherlands.

Vignat, F. and Villeneuve, F. (2007) 'Simulation of the manufacturing process, generation of a model of the manufactured parts', in US, Springer (Ed.): Digital Enterprise Technology, pp.545-552.

Villeneuve, F., Legoff, O. and Landon, Y. (2001) 'Tolerancing for manufacturing: a three-dimensional model', International Journal of Production Research, Vol. 39, No. 8 , pp.1625-1648.

Wolfram, S. (1983) 'Statistical mechanics of cellular automata', Review of Modern Physics, Vol. 55, pp.601-644.

Yin, X., Lee, S., Chen, W., Liu, W.K. and Horstemeyer, M.F. (2009) 'Efficient random field uncertainty propagation in design using multiscale analysis', Journal of Mechanical Design, Vol. 131, pp.021006-021010.

Yu, J-C. and Ishii, K. (1998) 'Design for robustness based on manufacturing variation patterns', Journal of Mechanical Design, Vol. 120, pp.196-202.

Zhou, S., Huang, Q. and Shi, J. (2003) 'State Space modeling of dimensional variation propagation in multistage machining process using differential motion vectors', IEEE Transactions on Robotics and Automation, Vol. 19, No. 2, pp.296-309.

Zhu, J. and Ting, K-L. (2001) 'Performance distribution analysis and robust design', Journal of Mechanical Design, Vol. 123, pp.11-17. 